\title{
Image features of spinning regular black holes based on a locality principle
}

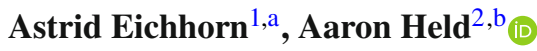 \\ ${ }^{1}$ CP3-Origins, University of Southern Denmark, Campusvej 55, 5230 Odense M, Denmark \\ 2 Theory Group, Blackett Laboratory, Imperial College London, London SW7 2AZ, UK
}

Received: 8 July 2021 / Accepted: 2 October 2021 / Published online: 21 October 2021

(C) The Author(s) 2021

\begin{abstract}
To understand the true nature of black holes, fundamental theoretical developments should be linked all the way to observational features of black holes in their natural astrophysical environments. Here, we take several steps to establish such a link. We construct a family of spinning, regular black-hole spacetimes based on a locality principle for new physics and analyze their shadow images. We identify characteristic image features associated to regularity (increased compactness and relative stretching) and to the locality principle (cusps and asymmetry) that persist in the presence of a simple analytical disk model. We conjecture that these occur as universal features of distinct classes of regular black holes based on different sets of construction principles for the corresponding spacetimes.
\end{abstract}

General Relativity (GR) has passed many observational tests $[1,2]$. In particular, it describes spacetime outside the event horizon of a black hole consistently with observations within the statistical and systematic uncertainties [37]. Despite these successes, a problem with the theoretical description of black holes in GR remains. The theory predicts a singularity of the space-time curvature, signalling its own breakdown. Thus, we do not understand the true nature of black holes. We know from observations that compact objects like black holes exist [8]. We know from theoretical consistency that these objects must be nonsingular. A two-step development is called for: theoretically consistent, regular spinning black-hole spacetimes are constructed with a subsequent extraction of image features relevant for the Event Horizon Telescope (EHT) [6,9-13] and observables accessible to other observations, e.g., [14-19].

The theoretical explorations of shadow images beyond GR mostly follow two distinct strategies.

In the first strategy, black-hole solutions are derived in gravity theories beyond GR [18,20-36]. This strategy has

\footnotetext{
${ }^{a}$ e-mail: eichhorn@cp3.sdu.dk

b e-mail: a.held@imperial.ac.uk (corresponding author)
}

two main disadvantages: First, it is impossible to cover every conceivable theory beyond GR. Second, the shadow-images from many distinct theories are bound to be indistinguishable given the finite resolution of the EHT.

In the second strategy, all possible deviations from a Kerr metric are parametrized without reference to a fundamental theory [37-43]. Thus, even a detection of a deviation would not provide any direct information on the nature of new gravitational physics.

In this paper, we develop a third strategy that lies inbetween the other two. We develop a parameterized family of black-hole metrics that is based on a set of physical principles. Our principled-parameterized strategy results in image features. We expect that these capture universal imprints of a class of fundamental theories.

To develop this new strategy, we propose a family of space-time metrics for spinning, regular black holes based on a locality principle. We derive observational signatures in their shadows that are characteristic imprints of the locality principle. Our analysis accounts for observationally relevant parameters like spin and inclination and includes a simple analytical disk model following [44].

In order to take steps linking fundamental theory to future observational data, we (i) start from a fundamental theoretical principle, (ii) construct a family of spacetime metrics based on it, and (iii) calculate images in a toy model for the astrophysical environment of the black hole.

Four principles for black-hole spacetimes In contrast to parameterized deviations from the Kerr spacetime, see, e.g., [37-43], we base our construction on four fundamental physical principles. We demand that

(i) the spacetime is described by a metric that is a solution to some dynamics beyond GR,

(ii) the metric has a correct Newtonian limit,

(iii) the spacetime is nonsingular everywhere, 
(iv) the local curvature in Kerr spacetime determines the magnitude of deviations (locality principle).

The last principle distinguishes our construction from other work in spinning spacetimes, e.g., [18,22,23,25,26,28,29, 32,34-36]. As we will see, its implementation results in distinct new spacetime and image features. It follows from a (classical or quantum) effective field theory point of view on new physics which implies that deviations from GR set in beyond a critical value of the local curvature. It is motivated by several research lines, where quantum or classical modifications of GR follow effective field theory principles, both for black holes, e.g., [45-52] and beyond, e.g., [5361]. Within the highly restricted setting of spherical symmetry, principles (i)-(iv) have been successfully implemented, cf. [62-66]. However, astrophysical black holes typically spin [7,67-69]. Yet, to the best of our knowledge, no example implements principle iv) for a spinning black hole. This is rooted in the intricate curvature structures of Kerr black holes: for instance, the Kretschmann scalar changes its sign at various locations throughout the spacetime. This makes it technically challenging to define an invariant local curvature scale as a new-physics scale.

For our construction, we require a coordinate invariant characterization of space-time curvature. We follow [70,71], provide a comprehensive overview of our construction in an extensive companion paper [72] and restrict ourselves to stating the result here: The local curvature for a Kerr black hole (with mass $m$ and spin parameter $a$ ) is characterized by the invariant

$K_{\mathrm{GR}}=\frac{48 m^{2}}{\left(r^{2}+a^{2} \cos ^{2}(\theta)\right)^{3}}$,

which provides an envelope to the absolute value of the maximum of all independent non-derivative curvature invariants of the Kerr spacetime. These independent curvature invariants are the Kretschmann scalar, $R_{\mu \nu \kappa \lambda} R^{\mu \nu \kappa \lambda}$, and $1 / 2 \epsilon_{\mu \nu \rho \sigma} R^{\rho \sigma \kappa \lambda} R_{\kappa \lambda}{ }^{\mu \nu}$. Independently, each of these is a non-monotonic function of the spacetime coordinates. Equation (1) provides a monotonic (i.e., monotonically decreasing with $r$ and monotonically rising from $\theta=0$ to $\theta=\pi / 2$ ) envelope of the maximum of the invariants. This expression is the basis to implement the physical idea that modifications of GR set in whenever any one of these curvature invariants is larger than the new-physics scale. Here, we set $G=1$ and choose ingoing Kerr coordinates $u, r, \theta, \phi$. We denote the mass and spin parameter by $m$ and $a$, respectively. To implement principle iv), deviations from Kerr must be functions of $K_{\mathrm{GR}} \cdot \ell_{\mathrm{NP}}^{4}$ only, where $\ell_{\mathrm{NP}}$ parameterizes the scale of new physics, which is unknown and treated as a free parameter in the following. To implement principles ii) and iii), we introduce an effective mass function $M\left(K_{\mathrm{GR}}\right)=M(r, \theta)$ which substitutes the constant mass in the Kerr metric, i.e.,

$$
\begin{aligned}
d s^{2}= & -\frac{r^{2}-2 M(r, \theta) r+a^{2} \cos ^{2}(\theta)}{r^{2}+a^{2} \cos ^{2}(\theta)} d u^{2}+2 d u d r \\
& -4 \frac{M(r, \theta) a r}{r^{2}+a^{2} \cos ^{2}(\theta)} \sin ^{2}(\theta) d u d \phi \\
& -2 a \sin ^{2}(\theta) d r d \phi+\left(r^{2}+a^{2} \cos ^{2}(\theta)\right) d \theta^{2} \\
& +\frac{\sin ^{2}(\theta)}{r^{2}+a^{2} \cos ^{2}(\theta)}\left[\left(a^{2}+r^{2}\right)^{2}\right. \\
& \left.-a^{2}\left(r^{2}-2 M(r, \theta) r+a^{2}\right) \cdot \sin ^{2}(\theta)\right] d \phi^{2} .
\end{aligned}
$$

The mass function must interpolate between $M\left(K_{\mathrm{GR}} \rightarrow\right.$ $0) \rightarrow m$ and $M\left(K_{\mathrm{GR}} \rightarrow \infty\right) \rightarrow 0$ and fall off faster than $\mathcal{O}\left(\left(K_{\mathrm{GR}} \cdot \ell_{\mathrm{NP}}^{4}\right)^{-1 / 2}\right)$ in order to ensure principle (ii) and (iii). Except for these two limits and the requirement of being nowhere singular, $M\left(K_{\mathrm{GR}}\right)$ can, in principle, be chosen freely to construct a model. A specific assumption for the dynamics of the new physics would provide additional constraints on $M\left(K_{\mathrm{GR}}\right)$.

Nevertheless, the locality principle implies that $M(r=$ const, $\theta)$ is smallest at $\theta=\pi / 2$, i.e., the modifications are largest in the equatorial plane. Across different choices of mass functions (which are monotonic functions of $K_{\mathrm{GR}}$ ), this results in universal observational features, as we will see by studying distinct examples,

$$
\begin{aligned}
M_{\mathrm{alg}}\left(K_{\mathrm{GR}}\right) & =\frac{m}{1+\ell_{\mathrm{NP}}^{4} K_{\mathrm{GR}}}, \\
M_{\exp }\left(K_{\mathrm{GR}}\right) & =m e^{-\left(\ell_{\mathrm{NP}}^{4} K_{\mathrm{GR}}\right)^{1 / 6}} .
\end{aligned}
$$

$M_{\text {alg }}$ reduces to a generalization of the Hayward mass function [64] and $M_{\text {exp }}$ to the Simpson-Visser mass function [66] in the spherically symmetric limit. For comparison, we choose a non-local model violating assumption (iv),

$$
M_{\text {non-local }}(r)=\frac{m}{1+\ell_{\mathrm{NP}}^{4} K_{\mathrm{GR}}\left(r, \theta=\frac{\pi}{2}\right)}=\frac{m}{1+\frac{48 m^{2} \ell_{\mathrm{NP}}^{4}}{r^{6}}} .
$$

For a Kerr black hole, $M\left(K_{\mathrm{GR}}\right)=m=$ const.

Similar mass functions (typically in the spherically symmetric limit) arise in the context of Loop Quantum Gravity, see, e.g. [24,27,31], asymptotic safety, see, e.g. [20,22,25, $30,65,73]$, non-commutative geometry, see, e.g. [21], and string theory, see, e.g. [33]. In [74], a related mass function was proposed in axisymmetry in the asymptotic-safety approach. Moreover, [75] links such regular black spacetimes to classical modified gravity theories; and [63] show that similar metrics arise in GR coupled to nonlinear electrodynamics.

In $[76,77]$, it has been pointed out that the inner horizon of such black hole metrics might be unstable. To analyze this potential instability, we would require a fundamental 
dynamics, which the nature of our principled-parameterized approach to black-hole images does not provide. Instead, this question may be addressed in individual gravity theories beyond GR.

A dent from local new physics To characterize the black-hole spacetime, we determine the location of the event horizon numerically, [72], cf. left-hand panel in Fig. 1 which highlights two features.

(i) Globally, the horizon shrinks compared to a singular Kerr black hole with the same $m$ and $a$. The same effect occurs in many spherically symmetric examples, see, e.g., [31,62,64-66, 74, 78,79] and for all three mass functions, including $M_{\text {non-local }}(r)$. This effect can be traced back to the singularity-resolving new physics which acts as a weakening of gravity leading to a more compact event horizon.

(ii) An additional effect is only present in the axisymmetric case: the event horizon features a dent at $\theta=\pi / 2$, cf. left-hand panel in Fig. 1, i.e., a minimum of the radius of the horizon as a function of $\theta$. The dent is a result of increased compactness in the equatorial plane. This follows from the angular dependence of the mass function $M\left(K_{\mathrm{GR}}\right)$ as a result of the classical curvature invariant $K_{\mathrm{GR}}$ in Eq. (1).

In contrast, the non-local mass function in Eq. (5) features no angular dependence and thus results in a spherically symmetric event horizon. More generally, this holds for any mass function which is independent of $\theta$, thus violating our locality principle.
Impact of astrophysical environments The typical environments of astrophysical black holes are accretion disks. We consider a simple model of such an environment by adding a simple model of a non-dynamical disk. We neglect absorptivity and assume that the disk is optically thin. There are strong indications that accretion disks of supermassive black holes such as M87* and Sgr A* could indeed be optically thin [80]. Finally, given the nearly monochromatic nature of present EHT observations, we focus on a single frequency $v$. In conclusion, we follow [44] and model the disk by a density function with variable disk parameter $h$, i.e.,

$n(r, \theta)=n_{0} \times \exp \left[-\frac{1}{2}\left(\frac{r^{2}}{100}+h^{2} \cos ^{2}(\theta)\right)\right]$.

In this setup, the radiative transfer equation for the intensity $I$ reduces to:

$\frac{d}{d \lambda}\left(\frac{I_{v}}{v^{3}}\right)=C n\left(x^{\mu}(\lambda)\right)$,

where $\lambda$ is the affine parameter and the radiative transfer equation is evaluated on the photon world line $x^{\mu}(\lambda) . C$ is a constant that, together with the density $n_{0}$, drops out once we normalize the intensity in the images. Finally, the radial null geodesics are obtained by numerically solving the geodesic equation. The impact of varying disk parameter $h$ on image features is studied in the longer companion paper [72].

Overall image difference The shadow of Kerr black holes deviates from a spherical shape by about $13 \%$ on the prograde side of the image. On this side, light rays are pulled closer to the black hole by frame dragging. The same effect exists for our regular black holes. Thus the images of regular spinning black holes show a similar flattening of the
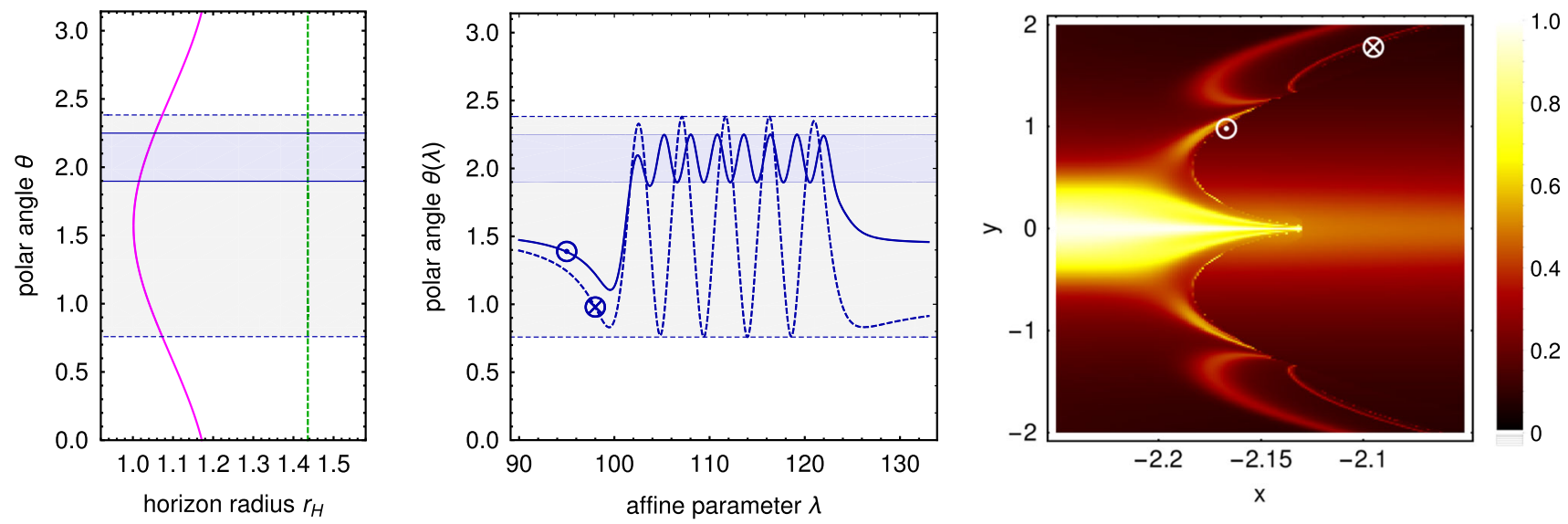

Fig. 1 Left-hand panel: parametric curves of the spherical Kerr horizon (dashed green) and the non-spherical horizon of the local, regular black-hole $\left(M_{\exp }\right.$ with $\left.\ell_{\mathrm{NP}}=0.2556 m \approx \ell_{\mathrm{NP}, \text { crit }}\right)$, both at spin parameter $a=0.9 \mathrm{~m}$. Middle panel: Different explicit trajectories (as a function of affine parameter $\lambda$ ) initiated at image points on the shadow boundary (marked in the right-hand panel) probing symmetric (dashed) and asym- metric (continuous) sections of the spacetime. Right-hand panel: The resulting intensity image (normalized to the brightest image point) for inclination $\theta_{\mathrm{obs}}=\pi / 2$. Cusps in the shadow boundary occur whenever trajectories jump between symmetric and asymmetric $\theta$-oscillations (cf. middle panel) 
shadow boundary and look largely comparable to the Kerr case, cf. Fig. 2. Given the finite resolution of the EHT, our study suggests that the imaged object $[6,9-13]$ could be a regular black hole satisfying our four principles.

This motivates the question whether future observations could, in principle, detect imprints of regularity and the locality principle. We compare to the case of a non-local mass function (cf. Eq. (5)) to tentatively classify which image features are associated to regularity and which to locality.

From Fig. 2, where we compare intensities in the Kerr and a regular case, the prograde image-side emerges as the interesting image location, which is why we focus on it in the following.

Shift of the prograde shadow boundary The shadow is more compact for regular than for Kerr black holes due to the more compact horizon. The effect is significantly larger on the prograde side, where frame dragging enables light rays to probe the geometry closer to the more compact horizon. The resulting appreciable shift of the prograde shadow boundary is not tied to the locality principle, see, e.g., $[18,34,78,81-$ 87].

This increase in compactness is not degenerate with the flattening effect of increased spin parameter $a$ in the Kerr case. The overall shape of the shadow boundary cannot be reproduced by any Kerr geometry, see [72].

Relative stretching Gravitational lensing in the vicinity of the black-hole horizon results in multiple lensed images of the disk. Corresponding image features can vary significantly with inclination but are visible at all inclinations, cf. Fig. 3 . This includes the M87* case, where the spin axis nearly points towards the observer, i.e., $\theta_{\mathrm{obs}} \approx 17 \pi / 180$, [13]. The distance between these features is stretched out in the case of regular black holes. The intensity peaks furthest away from the shadow boundary lie at similar locations in the image plane in the singular and regular case, cf. Fig. 2. As null
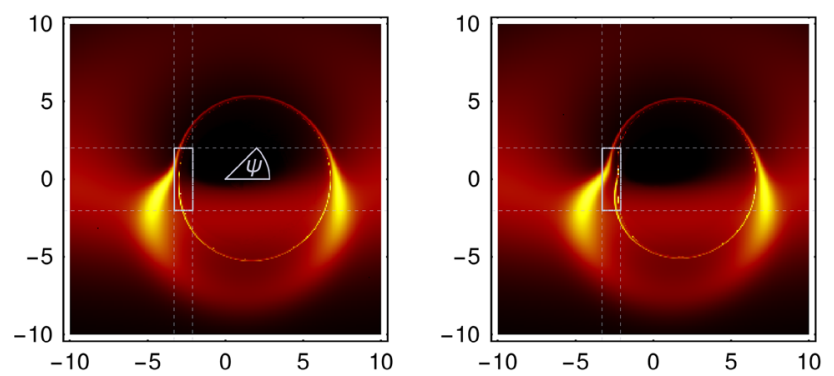

Fig. 2 Relative intensity in the $(x, y)$ image plane, normalized to the brightest image point (of both images), cf. color legend in Fig. 1. Left: Kerr black hole; Right: regular black hole with $M_{\exp }$ with $\ell_{\mathrm{NP}}=$ $0.2556 \mathrm{~m} \approx \ell_{\mathrm{NP}, \text { crit }}$. We choose $a=0.9 \mathrm{~m}$, inclination $\theta_{\mathrm{obs}}=8 \pi / 20$, and disk parameter $h=10 \mathrm{~m}$ in both panels. The gray boxes indicate the prograde region in which the deviations from Kerr spacetime are the largest, cf. Fig. 3 for detail images geodesics move towards the light sphere, the compactness of the probed geometry increases. Therefore, the distance to further inwards lying intensity maxima is stretched out, in comparison to the Kerr case.

The amount of stretching is a function of the radial and angular dependence of the mass function $M(r, \theta)$. In particular, $M_{\text {exp }}$ leads to a larger effect compared to $M_{\text {alg }}$. In both cases, the amount of stretching varies significantly with image angle $\psi$, because the mass function also depends on $\theta$. Therefore the increase in compactness has an angular $(\psi)$ dependence. In contrast, for a non-local mass function, the effect is more homogeneous across different image angles because the increase in compactness only depends on the radius.

Away from the shadow boundary, image features depend on the geometry and on the emission structure [88-90], see [72] for a first study of the impact of varying disk parameter $h$.

Cusps from the locality principle The overall shift and the stretching of image features distinguish regular from Kerr black holes, but are present for mass functions that either satisfy or violate the locality principle. This motivates us to search for potential distinguishing features between the two classes of regular mass functions. A characteristic difference between the two classes of geometries is the dent in the horizon, exclusively present for mass functions satisfying the locality principle. Indeed, we find that the latter can result in characteristic cusp-like features in the shadow images, cf. Fig. 3. These cusps are discontinuities in the shadow boundary. Just like shift and relative stretching, cusps are enhanced for $M_{\exp }$ compared to $M_{\mathrm{alg}}$. Moreover, they are most distinct when the regular black holes are viewed faceon $\left(\theta_{\text {obs }}=\pi / 2\right)$.

To understand the origin of the cusps, one needs to know that geodesics making up the shadow boundary cover bounded ranges in $\theta$ while orbiting the black hole [91-93]. The range between $\theta_{\min }(\psi)$ and $\theta_{\max }(\psi)$ depends on the image angle $\psi$. When the range of $\theta$ changes, the corresponding radius of the horizon differs, cf. left panel in Fig 1. Thus, null geodesics that arrive at distinct $\psi$ effectively probe different sections of the near-horizon geometry. For instance, trajectories on two sides of a cusp probe symmetric and asymmetric sections of the horizon geometry, respectively, cf. middle panel in Fig. 1. Discontinuous jumps in $\theta_{\min / \max }(\psi)$ result in discontinuities in the shadow boundary at $\psi$.

In particular, $\psi=\pi$ is associated to a particularly pronounced discontinuity at $\theta_{\mathrm{obs}} \approx \pi / 2$, cf. right-hand panel in Fig. 1. In this case, the dent in the event horizon is mirrored by a corresponding dent in the image, see also [74].

In contrast, for a spherically symmetric horizon, any section of the near-horizon geometry contains the same information on a constant horizon radius. Therefore changes in 

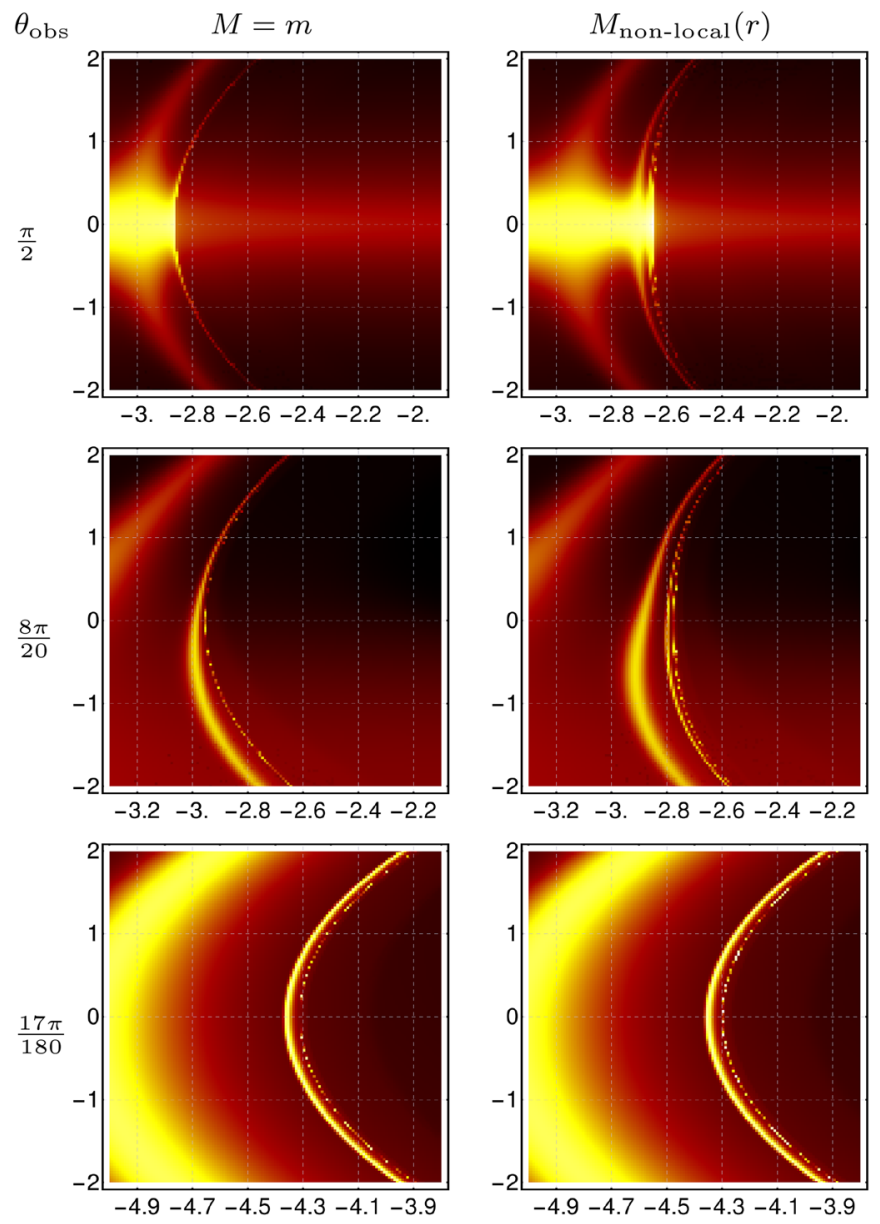

Fig. 3 Detailed intensity images of the prograde side of spinning black holes $(a=0.9 \mathrm{~m})$ in the $(x, y)$ image plane, normalized to the brightest image point (of all four images in each row), cf. color legend in Fig. 1. Columns from left to right correspond to mass functions $M=m$ (Kerr), $M_{\text {non-local }}(r)$ (cf. Eq. (5)), $M_{\text {alg }}(r, \theta)$ (cf. Eq. (3)), and $M_{\exp }(r, \theta)$ (cf. Eq. (4)), respectively. Apart from the Kerr case, the black-hole spacetimes are regular everywhere and, as in Fig. $2, \ell_{\mathrm{NP}}$ is

$\theta_{\min / \max }$ as a function of $\psi$ do not lead to discontinuities in the shadow boundary. This holds for singular Kerr black holes and any non-local mass function that is associated with a spherically symmetric event horizon.

Broken reflection symmetry from the locality principle From the images in Fig. 3, we observe that the lack of reflection symmetry about the $y=0$ axis generally characterizes images which are not face on, i.e., for which $\theta_{\text {obs }} \neq \pi / 2$. For a Kerr black hole, the asymmetry decreases towards and vanishes at the shadow boundary. The same observation holds for the non-local mass function, but not for a local mass function. For $M_{\mathrm{alg}}$ and $M_{\mathrm{exp}}$, reflection symmetry of the shadow boundary is broken. This is another consequence of an event horizon that breaks spherical symmetry. In fact, intuition based on geometric optics suggests that when view-
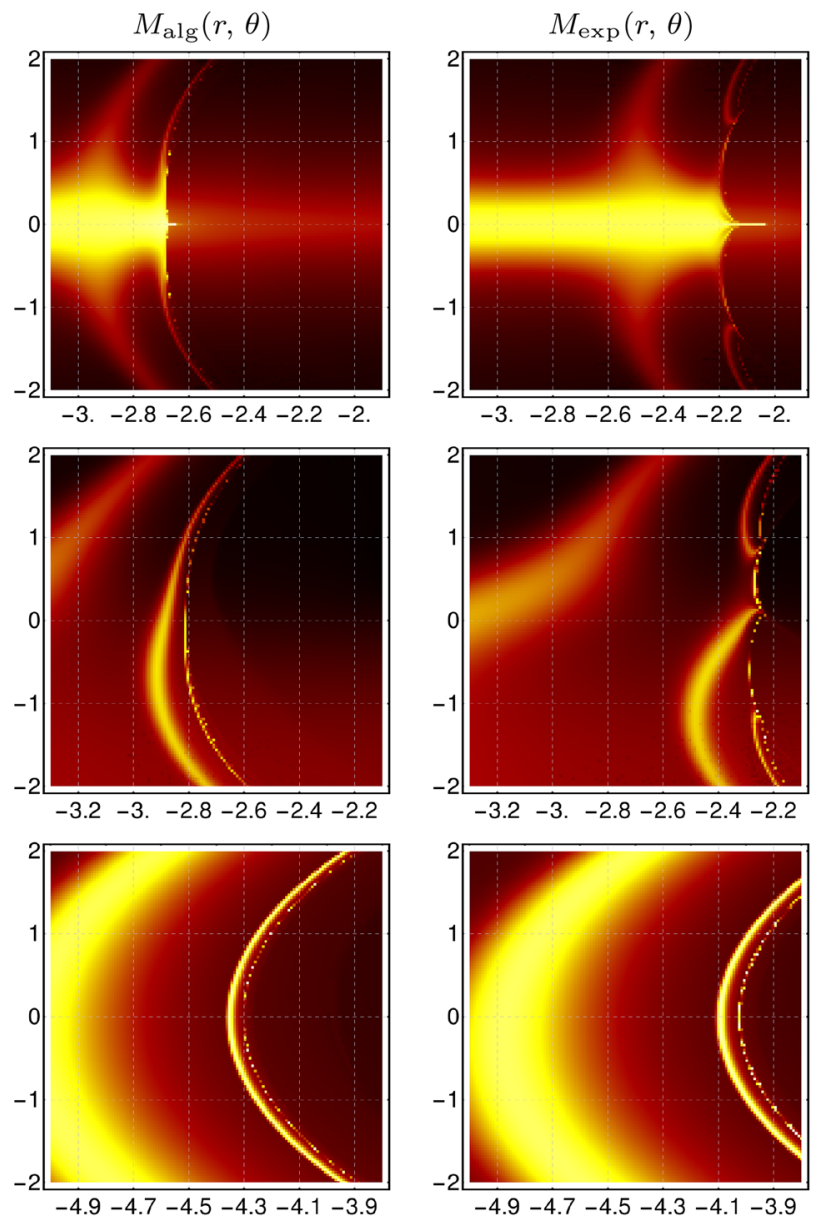

chosen close (i.e., with 4-digit precision) to the critical value $\ell_{\mathrm{NP}, \text { crit }}$ for which, in the respective spherically-symmetric case, the event horizon disappears. The latter maximizes the potential new-physics effect in the presence of an event horizon. The different rows depict different inclinations between the vector pointing from the black hole to the observer and the black hole's spin axis, i.e., $\theta_{\mathrm{obs}}=\frac{\pi}{2}, \frac{8 \pi}{20}, \frac{17 \pi}{180}$ from top to bottom

ing a non-spherically symmetric object, the resulting image depends on whether the object is tilted towards or away from the observer. Even if the object has a reflection symmetry about its equatorial plane, images at nontrivial inclination break this symmetry. This intuition describes the impact of the inclination on the shape of the shadow boundary.

Again, as for the cusp-like features, the asymmetry follows from the dent in the event horizon and hence our locality assumption.

Outlook: universality classes of new-physics principles Our results suggest that regular black holes could be characterized by a set of physical principles resulting in qualitatively distinct image features, constituting universality classes for black-hole shadows. A regularity principle results in a more compact shadow, a shifted prograde shadow boundary, and 
relative stretching of image features. All representatives of this principle that we have explored and others $[18,34,78,81-$ 87] exhibit these characteristics. Moreover, the locality principle could characterize a more constrained universality class with added image features, namely cusps and broken reflection symmetry.

We tentatively conjecture that a distinction of mass functions within a given universality class is rather difficult to achieve given finite EHT resolution capabilities, see however [94,95]. However, a distinction between such universality classes might potentially be achievable in the case of

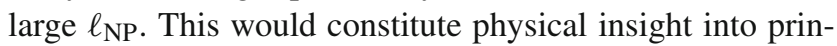
ciples underlying a more fundamental theory of gravity than GR.

These findings motivate a number of upgrades of our study, most importantly a fully dynamical accretion disk with more complete modelling of absorptivity and emissivity, as in [96].

Acknowledgements We gratefully acknowledge discussions with R. Gold. A. E. is supported by a research grant (29405) from VILLUM fonden. A. H. is supported by a Royal Society Newton International Fellowship NIF|R1\191008.

Data Availability Statement This manuscript has no associated data or the data will not be deposited. [Authors' comment: All data can be made available upon request.]

Open Access This article is licensed under a Creative Commons Attribution 4.0 International License, which permits use, sharing, adaptation, distribution and reproduction in any medium or format, as long as you give appropriate credit to the original author(s) and the source, provide a link to the Creative Commons licence, and indicate if changes were made. The images or other third party material in this article are included in the article's Creative Commons licence, unless indicated otherwise in a credit line to the material. If material is not included in the article's Creative Commons licence and your intended use is not permitted by statutory regulation or exceeds the permitted use, you will need to obtain permission directly from the copyright holder. To view a copy of this licence, visit http://creativecomm ons.org/licenses/by/4.0/.

Funded by SCOAP ${ }^{3}$.

\section{References}

1. C.M. Will, Living Rev. Relativ. 17, 4 (2014). https://doi.org/10. 12942/lrr-2014-4

2. E. Berti et al., Class. Quantum Gravity 32, 243001 (2015). https:// doi.org/10.1088/0264-9381/32/24/243001

3. B.P. Abbott et al., Phys. Rev. Lett. 116(22), 221101 (2016). https:// doi.org/10.1103/PhysRevLett.116.221101 [Erratum: Phys. Rev. Lett. 121, 129902 (2018)]

4. A. Hees et al., Phys. Rev. Lett. 118(21), 211101 (2017). https://doi. org/10.1103/PhysRevLett.118.211101

5. B.P. Abbott et al., Phys. Rev. D 100(10), 104036 (2019). https:// doi.org/10.1103/PhysRevD.100.104036

6. E.H.T. Collaboration, Astrophys. J. 875(1), L6 (2019). https://doi. org/10.3847/2041-8213/ab1141

7. R. Abbott et al. (2020). https://doi.org/10.1103/PhysRevD.103. 122002
8. V. Cardoso, P. Pani, Living Rev. Relativ. 22(1), 4 (2019). https:// doi.org/10.1007/s41114-019-0020-4

9. E.H.T. Collaboration, Astrophys. J. 875(1), L1 (2019). https://doi. org/10.3847/2041-8213/ab0ec7

10. E.H.T. Collaboration, Astrophys. J. 875(1), L2 (2019). https://doi. org/10.3847/2041-8213/ab0c96

11. E.H.T. Collaboration, Astrophys. J. 875(1), L3 (2019). https://doi. org/10.3847/2041-8213/ab0c57

12. E.H.T. Collaboration, Astrophys. J. 875(1), L4 (2019). https://doi. org/10.3847/2041-8213/ab0e85

13. E.H.T. Collaboration, Astrophys. J. 875(1), L5 (2019). https://doi. org/10.3847/2041-8213/ab0f43

14. A. Flachi, J.P.S. Lemos, Phys. Rev. D 87(2), 024034 (2013). https:// doi.org/10.1103/PhysRevD.87.024034

15. R. Carballo-Rubio, F. Di Filippo, S. Liberati, M. Visser, Phys. Rev. D 101, 084047 (2020). https://doi.org/10.1103/PhysRevD. 101.084047

16. G. Panotopoulos, A. Rincón, Eur. Phys. J. Plus 134(6), 300 (2019). https://doi.org/10.1140/epjp/i2019-12686-x

17. B. Zhou, A.B. Abdikamalov, D. Ayzenberg, C. Bambi, S. Nampalliwar, A. Tripathi, JCAP 01, 047 (2021). https://doi.org/10.1088/ 1475-7516/2021/01/047

18. C. Liu, T. Zhu, Q. Wu, K. Jusufi, M. Jamil, M. Azreg-Aïnou, A. Wang, Phys. Rev. D 101(8), 084001 (2020). https://doi.org/10. 1103/PhysRevD.101.084001

19. I. Agullo, V. Cardoso, A. del Rio, M. Maggiore, J. Pullin, Phys. Rev. Lett. 126(4), 041302 (2021). https://doi.org/10.1103/PhysRevLett. 126.041302

20. A. Bonanno, M. Reuter, Phys. Rev. D 62, 043008 (2000). https:// doi.org/10.1103/PhysRevD.62.043008

21. P. Nicolini, Int. J. Mod. Phys. A 24, 1229 (2009). https://doi.org/ 10.1142/S0217751X09043353

22. M. Reuter, E. Tuiran, Phys. Rev. D 83, 044041 (2011). https://doi. org/10.1103/PhysRevD.83.044041

23. C. Bambi, L. Modesto, Phys. Lett. B 721, 329 (2013). https://doi. org/10.1016/j.physletb.2013.03.025

24. R. Gambini, J. Pullin, Phys. Rev. Lett. 110(21), 211301 (2013). https://doi.org/10.1103/PhysRevLett.110.211301

25. D.F. Litim, K. Nikolakopoulos, JHEP 04, 021 (2014). https://doi. org/10.1007/JHEP04(2014)021

26. M. Azreg-Aïnou, Phys. Rev. D 90(6), 064041 (2014). https://doi. org/10.1103/PhysRevD.90.064041

27. H.M. Haggard, C. Rovelli, Phys. Rev. D 92(10), 104020 (2015). https://doi.org/10.1103/PhysRevD.92.104020

28. B. Toshmatov, B. Ahmedov, A. Abdujabbarov, Z. Stuchlik, Phys. Rev. D 89(10), 104017 (2014). https://doi.org/10.1103/PhysRevD. 89.104017

29. S.G. Ghosh, S.D. Maharaj, Eur. Phys. J. C 75, 7 (2015). https://doi. org/10.1140/epjc/s10052-014-3222-7

30. J.M. Pawlowski, D. Stock, Phys. Rev. D 98(10), 106008 (2018). https://doi.org/10.1103/PhysRevD.98.106008

31. A. Ashtekar, J. Olmedo, P. Singh, Phys. Rev. Lett. 121(24), 241301 (2018). https://doi.org/10.1103/PhysRevLett.121.241301

32. R. Kumar, B.P. Singh, S.G. Ghosh, Ann. Phys. 420, 168252 (2020). https://doi.org/10.1016/j.aop.2020.168252

33. P. Nicolini, E. Spallucci, M.F. Wondrak, arXiv preprint (2019). https://doi.org/10.1016/j.physletb.2019.134888

34. E. Contreras, A. Rincón, G. Panotopoulos, P. Bargueño, B. Koch, Phys. Rev. D 101(6), 064053 (2020). https://doi.org/10.1103/ PhysRevD.101.064053

35. H.C.D.L. Junior, L.C.B. Crispino, P.V.P. Cunha, C.A.R. Herdeiro (2021). https://doi.org/10.1103/PhysRevD.103.084040

36. J. Mazza, E. Franzin, S. Liberati (2021). https://doi.org/10.3390/ universe7030048

37. S.J. Vigeland, S.A. Hughes, Phys. Rev. D 81, 024030 (2010). https://doi.org/10.1103/PhysRevD.81.024030 
38. T. Johannsen, D. Psaltis, Phys. Rev. D 83, 124015 (2011). https:// doi.org/10.1103/PhysRevD.83.124015

39. S. Vigeland, N. Yunes, L. Stein, Phys. Rev. D 83, 104027 (2011). https://doi.org/10.1103/PhysRevD.83.104027

40. V. Cardoso, P. Pani, J. Rico, Phys. Rev. D 89, 064007 (2014). https:// doi.org/10.1103/PhysRevD.89.064007

41. T. Johannsen, Phys. Rev. D 88(4), 044002 (2013). https://doi.org/ 10.1103/PhysRevD.88.044002

42. N. Lin, N. Tsukamoto, M. Ghasemi-Nodehi, C. Bambi, Eur. Phys. J. C 75(12), 599 (2015). https://doi.org/10.1140/epjc/ s10052-015-3837-3

43. R. Konoplya, L. Rezzolla, A. Zhidenko, Phys. Rev. D 93(6), 064015 (2016). https://doi.org/10.1103/PhysRevD.93.064015

44. R. Gold, A.E. Broderick, Z. Younsi, C.M. Fromm, C.F. Gammie, M. Mościbrodzka, H.Y. Pu, T. Bronzwaer, J. Davelaar, J. Dexter et al., ApJ 897(2), 148 (2020). https://doi.org/10.3847/1538-4357/ ab96c6

45. N.E.J. Bjerrum-Bohr, J.F. Donoghue, B.R. Holstein, Phys. Rev. D 68, 084005 (2003). https://doi.org/10.1103/PhysRevD.68. 084005. https://doi.org/10.1103/PhysRevD.71.069904 [Erratum: Phys. Rev. D 71, 069904 (2005)]

46. H. Lu, A. Perkins, C.N. Pope, K.S. Stelle, Phys. Rev. Lett. 114(17), 171601 (2015). https://doi.org/10.1103/PhysRevLett.114.171601

47. V. Cardoso, M. Kimura, A. Maselli, L. Senatore, Phys. Rev. Lett. 121(25), 251105 (2018). https://doi.org/10.1103/PhysRevLett. 121.251105

48. R.A. Konoplya, A.F. Zinhailo, Eur. Phys. J. C 80(11), 1049 (2020). https://doi.org/10.1140/epjc/s10052-020-08639-8

49. J.N. Borissova, A. Eichhorn (2020). https://doi.org/10.3390/ universe7030048

50. C. de Rham, J. Francfort, J. Zhang, Phys. Rev. D 102(2), 024079 (2020). https://doi.org/10.1103/PhysRevD.102.024079

51. Y. Xie, J. Zhang, H.O. Silva, C. de Rham, H. Witek, N. Yunes (2021). https://doi.org/10.1103/PhysRevLett.126.241104

52. A. Held, H. Lim (2021). https://journals.aps.org/prd/accepted/ 97070Qe9Q381842178bb0346618a98b6bfcf68be2

53. A.A. Starobinsky, Adv. Ser. Astrophys. Cosmol. 3, 130 (1987). https://doi.org/10.1016/0370-2693(80)90670-X

54. I.G. Avramidi, A.O. Barvinsky, Phys. Lett. B 159, 269 (1985). https://doi.org/10.1016/0370-2693(85)90248-5

55. J.F. Donoghue, Phys. Rev. D 50, 3874 (1994). https://doi.org/10. 1103/PhysRevD.50.3874

56. T.P. Sotiriou, V. Faraoni, Rev. Mod. Phys. 82, 451 (2010). https:// doi.org/10.1103/RevModPhys.82.451

57. A. De Felice, S. Tsujikawa, Living Rev. Relativ. 13, 3 (2010). https://doi.org/10.12942/lrr-2010-3

58. L. Alvarez-Gaume, A. Kehagias, C. Kounnas, D. Lüst, A. Riotto, Fortsch. Phys. 64(2-3), 176 (2016). https://doi.org/10.1002/prop. 201500100

59. M. Levi, Rep. Prog. Phys. 83(7), 075901 (2020). https://doi.org/ 10.1088/1361-6633/ab12bc

60. A. Eichhorn, Front. Astron. Space Sci. 5, 47 (2019). https://doi. org/10.3389/fspas. 2018.00047

61. T. Kobayashi, Rep. Prog. Phys. 82(8), 086901 (2019). https://doi. org/10.1088/1361-6633/ab2429

62. I. Dymnikova, Gen. Relativ. Gravit. 24, 235 (1992). https://doi.org/ 10.1007/BF00760226

63. E. Ayon-Beato, A. Garcia, Phys. Rev. Lett. 80, 5056 (1998). https:// doi.org/10.1103/PhysRevLett.80.5056

64. S.A. Hayward, Phys. Rev. Lett. 96, 031103 (2006). https://doi.org/ 10.1103/PhysRevLett.96.031103

65. A. Platania, Eur. Phys. J. C 79(6), 470 (2019). https://doi.org/10. 1140/epjc/s10052-019-6990-2

66. A. Simpson, M. Visser, Universe 6(1), 8 (2019). https://doi.org/10. 3390/universe6010008
67. C.F. Gammie, S.L. Shapiro, J.C. McKinney, Astrophys. J. 602, 312 (2004). https://doi.org/10.1086/380996

68. C.S. Reynolds, Space Sci. Rev. 183(1-4), 277 (2014). https://doi. org/10.1007/s11214-013-0006-6

69. C.S. Reynolds, arXiv e-prints arXiv:2011.08948 (2020)

70. J. Carminati, R.G. McLenaghan, J. Math. Phys. 32(11), 3135 (1991). https://doi.org/10.1063/1.529470

71. E. Zakhary, C.B.G. McIntosh, Gen. Relativ. Gravit. 29(5), 539 (1997). https://doi.org/10.1023/A:1018851201784

72. A. Eichhorn, A. Held, JCAP 05, 073 (2021). https://doi.org/10. 1088/1475-7516/2021/05/073

73. A. Adeifeoba, A. Eichhorn, A. Platania, Class. Quantum Gravity 35(22), 225007 (2018). https://doi.org/10.1088/1361-6382/aae6ef

74. A. Held, R. Gold, A. Eichhorn, JCAP 1906, 029 (2019). https:// doi.org/10.1088/1475-7516/2019/06/029

75. O. Baake, C. Charmousis, M. Hassaine, M. San (2021). https://doi. org/10.1088/1475-7516/2021/06/021

76. R. Carballo-Rubio, F. Di Filippo, S. Liberati, M. Visser (2019), https://doi.org/10.1103/PhysRevD.101.084047

77. R.C. Rubio, F. Di Filippo, S. Liberati, C. Pacilio, M. Visser (2021) https://doi.org/10.1007/JHEP05(2021)132

78. M. Amir, S.G. Ghosh, Phys. Rev. D 94(2), 024054 (2016). https:// doi.org/10.1103/PhysRevD.94.024054

79. Z. Stuchlík, J. Schee, Eur. Phys. J. C 79(1), 44 (2019). https://doi. org/10.1140/epjc/s10052-019-6543-8

80. M.D. Johnson et al., Science 350(6265), 1242 (2015). https://doi. org/10.1126/science.aac7087

81. A. Abdujabbarov, M. Amir, B. Ahmedov, S.G. Ghosh, Phys. Rev. D 93(10), 104004 (2016). https://doi.org/10.1103/PhysRevD.93. 104004

82. N. Tsukamoto, Phys. Rev. D 97(6), 064021 (2018). https://doi.org/ 10.1103/PhysRevD.97.064021

83. H.M. Wang, Y.M. Xu, S.W. Wei, JCAP 03, 046 (2019). https://doi. org/10.1088/1475-7516/2019/03/046

84. I. Dymnikova, K. Kraav, Universe 5(7), 163 (2019). https://doi. org/10.3390/universe5070163

85. R. Shaikh, Phys. Rev. D 100(2), 024028 (2019). https://doi.org/10. 1103/PhysRevD.100.024028

86. R. Kumar, S.G. Ghosh, JCAP 07(07), 053 (2020). https://doi.org/ 10.1088/1475-7516/2020/07/053

87. S.G. Ghosh, M. Amir, S.D. Maharaj, Nucl. Phys. B 957, 115088 (2020). https://doi.org/10.1016/j.nuclphysb.2020.115088

88. A.E. Broderick, A. Loeb, Mon. Not. R. Astron. Soc. 367, 905 (2006). https://doi.org/10.1111/j.1365-2966.2006.10152.x

89. A.E. Broderick, V.L. Fish, M.D. Johnson, K. Rosenfeld, C. Wang, S.S. Doeleman, K. Akiyama, T. Johannsen, A.L. Roy, Astrophys. J. 820(2), 137 (2016). https://doi.org/10.3847/0004-637X/820/2/ 137

90. P. Tiede, H.Y. Pu, A.E. Broderick, R. Gold, M. Karami, J.A. Preciado-López (2020). https://doi.org/10.3847/1538-4357/ ab744c

91. S.E. Vazquez, E.P. Esteban, Nuovo Cim. B 119, 489 (2004). https:// doi.org/10.1393/ncb/i2004-10121-y

92. S.E. Gralla, A. Lupsasca, Phys. Rev. D 101(4), 044032 (2020). https://doi.org/10.1103/PhysRevD.101.044032

93. E. Himwich, M.D. Johnson, A. Lupsasca, A. Strominger, Phys. Rev. D 101(8), 084020 (2020). https://doi.org/10.1103/PhysRevD. 101.084020

94. L. Blackburn, et al. (2019). arxiv:1909.01411

95. D. Pesce, K. Haworth, G.J. Melnick, L. Blackburn, M. Wielgus, M.D. Johnson, A. Raymond, J. Weintroub, D.C.M. Palumbo, S.S. Doeleman, D.J. James, Bull. Am. Astron. Soc. 51, 176 (2019)

96. O. Porth et al., Astrophys. J. Suppl. 243(2), 26 (2019). https://doi. org/10.3847/1538-4365/ab29fd 